\title{
Cell-free hemoglobin mediated oxidative stress is associated with acute kidney injury and renal replacement therapy in severe falciparum malaria: an observational study
}

Katherine Plewes ${ }^{1,2,3}$, Hugh W.F. Kingston 1,2,4, Aniruddha Ghose ${ }^{5}$, Richard J. Maude ${ }^{1,2}$, M. Trent Herdman ${ }^{1}$, Stije J. Leopold ${ }^{1,2}$, Haruhiko Ishioka ${ }^{1}$, Md. Mahtab Uddin Hasan ${ }^{5}$, Md. Shafiul Haider ${ }^{6}$, Shamsul Alam ${ }^{7}$ Kim A. Piera ${ }^{4}$, Prakaykaew Charunwatthana ${ }^{1}$, Kamolrat Silamut ${ }^{1}$, Tsin W. Yeo ${ }^{4,8}$, Md. Abul Faiz ${ }^{9}$, Sue J Lee ${ }^{1,2}$, Mavuto Mukaka ${ }^{1,2}$, Gareth D.H. Turner ${ }^{1,2}$, Nicholas M. Anstey ${ }^{4}$, L. Jackson Roberts $\|^{10}$, Nicholas J. White ${ }^{1,2}$, Nicholas P.J. Day ${ }^{1,2}$, Md. Amir Hossain ${ }^{5}$ and Arjen M. Dondorp ${ }^{1,2^{*}}$

\begin{abstract}
Background: Intravascular hemolysis is an intrinsic feature of severe malaria pathophysiology but the pathogenic role of cell-free hemoglobin-mediated oxidative stress in severe malaria associated acute kidney injury (AKI) is unknown.

Methods: As part of a prospective observational study, enrolment plasma cell-free hemoglobin (CFH), lipid peroxidation markers ( $F_{2}$-isoprostanes ( $F_{2}-\mid$ soPs $)$ and isofurans $(I s o F s)$ ), red cell deformability, and serum creatinine were quantified in Bangladeshi patients with severe falciparum malaria $(n=107)$, uncomplicated malaria $(n=80)$ and sepsis $(n=28)$. The relationships between these indices and kidney function and clinical outcomes were examined.

Results: AKI was diagnosed at enrolment in 58\% (62/107) of consecutive patients with severe malaria, defined by an increase in creatinine $\geq 1.5$ times expected baseline. Severe malaria patients with AKI had significantly higher plasma cell-free hemoglobin (geometric mean CFH: $8.8 \mu \mathrm{M} ; 95 \% \mathrm{Cl}, 6.2-12.3 \mu \mathrm{M}), \mathrm{F}_{2}$-isoprostane $(56.7 \mathrm{pg} / \mathrm{ml} ; 95 \% \mathrm{Cl}$, $45.3-71.0 \mathrm{pg} / \mathrm{ml})$ and isofuran $(109.2 \mathrm{pg} / \mathrm{ml} ; 95 \% \mathrm{Cl}, 85.1-140.1 \mathrm{pg} / \mathrm{ml})$ concentrations on enrolment compared to

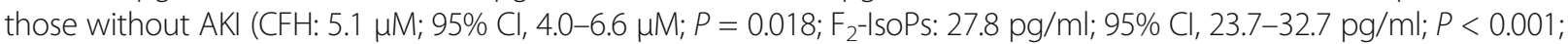
IsoFs: $41.7 \mathrm{pg} / \mathrm{ml} ; 95 \% \mathrm{Cl}, 30.2-57.6 \mathrm{pg} / \mathrm{ml} ; P<0.001)$. Cell-free hemoglobin correlated with markers of hemolysis, parasite burden (P. falciparum histidine rich protein 2 (PfHRP2)), and $F_{2}-I s O P s$. Plasma $F_{2}-I s o P s$ and IsoFs inversely correlated with $\mathrm{pH}$, positively correlated with creatinine, PfHRP2 and fractional excretion of sodium, and were higher in patients later requiring hemodialysis. Plasma $\mathrm{F}_{2}$-IsoP concentrations also inversely correlated with red cell deformability and were higher in fatal cases. Mixed effects modeling including an interaction term for CFH and time showed that $\mathrm{F}_{2}-\mathrm{IsOPs}$, IsoFs, PfHRP2, CFH, and red cell rigidity were independently associated with increasing creatinine over $72 \mathrm{~h}$. Multivariable logistic regression showed that admission $\mathrm{F}_{2}$-IsoPs, IsoFs and red cell deformability were associated with the need for subsequent hemodialysis.

(Continued on next page)
\end{abstract}

\footnotetext{
* Correspondence: arjen@tropmederes.ac

${ }^{1}$ Mahidol Oxford Tropical Medicine Research Unit, Faculty of Tropical

Medicine, Mahidol University, Bangkok, Thailand

${ }^{2}$ Centre for Tropical Medicine and Global Health, Nuffield Department of

Medicine, University of Oxford, Oxford, UK

Full list of author information is available at the end of the article
} 
(Continued from previous page)

Conclusions: Cell-free hemoglobin and lipid peroxidation are associated with acute kidney injury and disease severity in falciparum malaria, suggesting a pathophysiological role in renal tubular injury. Evaluation of adjunctive therapies targeting cell-free hemoglobin-mediated oxidative stress is warranted.

Keywords: Acute kidney injury, Pathophysiology, Falciparum malaria, Cell-free hemoglobin, Oxidative stress

\section{Background}

Severe falciparum malaria is characterized by intravascular hemolysis, where cell-free hemoglobin (CFH) increases with disease severity [1]. Sources of $\mathrm{CFH}$ include rupture of parasitized red blood cells (RBC) at schizogony, and destruction of uninfected erythrocytes, most prominently in patients with blackwater fever (hemoglobinuria) [2]. In 400 BC, Hippocrates first associated blackwater fever with anuria and mortality; findings that consistently resurfaced after Firth's report in 1886 [3, 4]. More recently, this condition of fulminant hemolysis has been associated with kidney dysfunction in up to $64 \%$ of patients [5], but the underlying mechanisms have not been fully characterized.

When the degree of intravascular hemolysis exceeds the scavenging capacity of plasma haptoglobin for hemoglobin, CFH dimers are filtered by the glomeruli and reabsorbed by the proximal tubule. Once the reabsorptive capacity is exceeded, hemoglobin appears in the urine [6]. CFH is independently associated with AKI in patients post-cardiopulmonary bypass, and with mortality in bacterial sepsis [7-9]. Hemoproteins, hemoglobin and myoglobin, are pathogenic as prooxidants when released heme is not scavenged by hemopexin. Heme redox cycling between ferric and ferryl states then generates globin radicals inducing lipid peroxidation [10]. In vivo studies on oxidative injury have been hampered by the paucity of stable and specific markers of oxidative stress.

$\mathrm{CFH}$-mediated non-enzymatic lipid peroxidation of arachidonic acid generates isomers of prostaglandins, $\mathrm{F}_{2}$-isoprostanes $\left(\mathrm{F}_{2}\right.$-IsoPs) and isofurans (IsoFs) [11, 12]. $\mathrm{F}_{2}$-IsoPs are generated at low oxygen tension whereas IsoFs are generated at higher oxygen tension and together are considered robust in vivo measures of oxidative stress $[11,12]$. In the current study, the hypothesis was that $\mathrm{CFH}$-mediated oxidative stress could cause renal damage either through a direct effect on renal tubules, through a reduction in red cell deformability (RCD), or through the vasoconstrictive properties of $\mathrm{F}_{2}$-IsoPs.

Arachidonic acids, such as red cell membrane phospholipids, are particularly vulnerable to free radicalmediated lipid peroxidation. Oxidative stress-induced reduction of RCD has been proposed to play a role in renal insufficiency [13]. In malaria, the high arachidonic acid content of infected RBC membranes reduces with intracellular parasite maturation, suggesting membrane peroxidation occurs during parasite development [14]. $\mathrm{F}_{2}$-IsoPs are considered not just bystanders of oxidative injury but are bioactive renal vasoconstrictors [12]. Both $\mathrm{F}_{2}$-IsoPs and IsoFs have been associated with AKI in patients with rhabdomyolysis and hemolysis postcardiopulmonary bypass [15-17]. Other plasma and urinary markers of oxidative stress have been shown to be significantly elevated in severe malaria compared to uncomplicated malaria [18-21].

The role of plasma CFH-mediated lipid peroxidation in the pathophysiology of severe malaria and AKI has not been described. Gaining a better understanding of the pathophysiology in malaria will help towards the development of targeted therapies. This study aimed to examine the generation of $\mathrm{CFH}$-mediated lipid peroxidation and its role in AKI and malaria severity by analyzing the associations between $\mathrm{CFH}, \mathrm{F}_{2}$-IsoPs, IsoFs, red cell deformability, and creatinine in patients with falciparum malaria.

\section{Methods}

\section{Study aim, design and setting}

The aim of this study was to assess CFH-mediated lipid peroxidation and its role in AKI and disease severity in falciparum malaria. This prospective observational study was conducted at Chittagong Medical College Hospital, Bangladesh from 2011 to 2014. This tertiary hospital receives referrals from malaria hypoendemic areas, and has basic facilities for intensive care and hemodialysis.

\section{Patient characteristics}

Patients admitted with slide confirmed severe or uncomplicated $P$. falciparum malaria were recruited upon diagnosis. Positive microscopy of peripheral blood required the presence of asexual stages of $P$. falciparum. Uncomplicated malaria was defined as asexual $P$. falciparum slide positivity without severity criteria. Criteria for severe malaria were: coma (Glasgow Coma Score < 11), shock (systolic blood pressure (SBP) $<80 \mathrm{mmHg}$ with cool extremities), anemia, (hematocrit $<20 \%$ plus parasitemia $>100,000 / \mu \mathrm{l}$ ), jaundice (total bilirubin $>51.3 \mu \mathrm{mol} / \mathrm{L}$ plus parasitemia $>100,000 / \mu \mathrm{l}$ ), hyperparasitemia (asexual parasitemia $>10 \%$ ), acidosis (bicarbonate $<15 \mathrm{mmol} / \mathrm{L}$ ), hyperlactatemia (lactate $>4 \mathrm{mmol} / \mathrm{L}$ ), hypoglycemia (glucose $<2.22 \mathrm{mmol} / \mathrm{L}$ ), convulsions ( $\geq 2$ in $24 \mathrm{~h}$ ), pulmonary edema, and/or AKI (serum creatinine $>3 \mathrm{mg} / \mathrm{dl}$ ). Patients 
were treated with parenteral artesunate (Guilin No.2 Pharmaceuticals, Guangxi, China) followed by artemether/lumefantrine (Novartis, Basel, Switzerland) and managed according to WHO treatment guidelines [22]. Hemodialysis was initiated according to local nephrologists. Indications for dialysis in this setting include: (1) anuria for more than $24 \mathrm{~h}$, (2) severe electrolyte and acid-base disturbance, (3) serum creatinine $>3 \mathrm{mg} / \mathrm{dl}$ with urine output $<0.5 \mathrm{ml} / \mathrm{kg} / \mathrm{h}$ for $12 \mathrm{~h}$, or (4) gradual rise in creatinine despite normal urine output. A control group of sepsis patients hospitalized with suspected bacterial infection and at least $2 / 4$ systemic inflammatory response criteria $(n=28)$ was also recruited [23]. Data from non-pregnant patients aged $\geq 10$ years is presented. Patients were followed until discharge or death; those with AKI were followed in hospital until renal recovery, if possible, as gauged by local nephrologists. Follow up after hospital discharge was challenging due to the distances patients travel to the teriary care center.

\section{Study procedures}

After enrolment, a medical history and physical examination were performed. Patients were seen 6-hourly until discharge or death. Enrolment venous blood samples were analyzed for electrolytes, glucose, $\mathrm{pH}$ and bicarbonate using a bedside analyzer (iSTAT, Abbott). Parasitemia was assessed 6-hourly from thick and thin smears until parasite clearance. Blood and urine for creatinine measurement were collected every $24 \mathrm{~h}$ for three days. Serum, heparinized plasma and urine were frozen in liquid nitrogen within one hour of collection. Serum creatinine was measured using an Olympus analyzer (Beckman Coulter Inc.).

\section{Assays}

Plasma and urine $\mathrm{F}_{2}$-isoprostane and isofuran concentrations were determined by gas chromatography-mass spectrometry at Vanderbilt University, as described $[11,24]$. The 24-h urinary excretion rate of urine $F_{2}$ isoprostane and isofuran concentrations were calculated as: concentration $\mathrm{x}$ volume $\times 24 \mathrm{~h} /$ time of collection [25]. Plasma CFH concentrations were measured by ELISA (Bethyl Laboratories), as described [1]. Plasma Plasmodium falciparum histidine rich protein 2 (PfHRP2), a biomarker of total parasite burden, was quantified using commercial sandwich ELISA (Celisa, Cellabs), as described [26].

Red cell deformability (RCD) was measured at enrolment using a laser-assisted optical rotational cell analyzer (LORCA, Mechatronic) immediately after blood collection $[27,28]$. The deformability was measured by ellipticity of RBCs and described by the elongation index; (long minus short axes lengths) divided by (long plus short axes lengths). RCD was assessed at shear stresses ranging from 0.3 to $30 \mathrm{~Pa}$. In capillaries, shear stresses of $1.7 \mathrm{~Pa}$ and above are encountered [29].

\section{Acute kidney injury}

Patients were classified according to AKI status at enrolment as defined by an increase in serum creatinine $\geq 1.5$ times expected baseline, known or presumed to have occurred within the prior seven days (as per the Kidney Disease Improving Global Outcomes (KDIGO) classification system) [30]. Since urine output was not available for all patients, and the duration of illness at presentation was always greater than $48 \mathrm{~h}$, these criteria were not incorporated for enrolment AKI diagnosis. As preadmission creatinine values were not available, expected baseline creatinine values were calculated as recommended using the Modification of Diet in Renal Disease formula assuming a glomerular filtration rate (GFR) of $75 \mathrm{ml} / \mathrm{min} / 1.73 \mathrm{~m}^{2}$ for participants 19 years and older [30] and using the Bedside Schwartz formula assuming a GFR of $100 \mathrm{ml} / \mathrm{min} / 1.73 \mathrm{~m}^{2}$ for those 18 years and younger [31-33]. The highest KDIGO AKI staging was assessed both on enrolment and during admission in order to accurately present the heterogeneous AKI status at the time of enrolment and subsequent kidney function during admission in those that survived. Stage 2 AKI was defined as an increase to $\geq 2.0-2.9$ times expected baseline; Stage 3 as either an increase to $\geq 3$ times expected baseline, an increase in creatinine to $\geq 4 \mathrm{mg} / \mathrm{dl}$, initiation of RRT, or in patients $<18$ years a decrease in GFR to $<35 \mathrm{ml} / \mathrm{min} / 1.73 \mathrm{~m}^{2}$ [30]. Creatinine used for enrolment AKI stratification were performed on samples drawn prior to hemodialysis.

\section{Statistical analysis}

Groups were compared using Wilcoxon rank-sum test or Student's $t$-tests depending on the distribution of the data. Correlations were assessed using Spearman's correlation coefficient. As the hypothesis was that $\mathrm{F}_{2}$-IsoPs, IsoFs, and CFH contribute to AKI, these pre-specified variables were assessed in a mixed effects model using creatinine as the dependent variable, and in a logistic regression model using hemodialysis as the dependant variable. As the $\mathrm{F}_{2}$-IsoPs and IsoFs were highly collinear $\left(r_{p}=0.67 ; p<0.001\right)$, their association with creatinine was assessed in separate multivariable models. The multivariable models included adjustment for age, SBP, PfHRP2 and RCD as these are considered physiologically relevant in contributing to AKI. In the mixed effect model, age, SBP, and time were modeled as fixed effects while the rest were treated as random effects. All prespecified and known AKI risk factor variables that were associated with creatinine were included in a multivariable mixed effects model. The interaction between enrolment $\mathrm{CFH}$ and time was also assessed. To account for hemodialysis confounding the decline in creatinine concentrations, creatinine values were adjusted at each time point following dialysis until time of death by using 
a creatinine rise of $1.5 \mathrm{mg} / \mathrm{dl}$ per day as proposed for anephric states [34]. Known risk factors for hemodialysis, including additional markers of disease severity (number of severity criteria, GCS, and lactate), were assessed using backward stepwise logistic regression including variables (Table 5) with a $p$-value of $<0.10$ on univariable analysis. Selection of the final model was based on the Akaike information criteria (AIC). Software used were STATA14.0 (Stata), and Prism 6 (Graphpad Software).

\section{Results}

\section{Baseline characteristics and clinical course in hospital}

A total of 107 consecutive patients with severe malaria were enrolled (Tables 1 and 2), as well as 80 patients with uncomplicated malaria and 28 with (suspected) bacterial sepsis as comparator control groups. Among patients with severe malaria, 58\% (62/107) had AKI on enrolment, while another $9 \%(10 / 107)$ subsequently developed AKI during admission (Table 1). The severity of AKI varied with 50\% (31/62) meeting the World Health Organization malaria guideline criteria for AKI (creatinine $>3 \mathrm{mg} / \mathrm{dl}$ ) [22], of whom $84 \%(26 / 31)$ were KDIGO stage 3 and $16 \%(5 / 31)$ had a further progression from KDIGO stage 2 to 3 during admission. In the AKI on enrolment group, 47\% (29/62) received hemodialysis; of whom $28 \%$ (8/29) died, and 53\% (33/62) did not receive hemodialysis; of whom $52 \%$ (17/33) died (OR for death without dialysis 2.8 (95\% CI, 0.9-9.4; $P=0.048)$. Among the $72 \%(21 / 29)$ survivors in the AKI group who received dialysis, the median renal recovery time was 21 days (IQR, $13-42$ days; $n=7$ ). Among the $48 \%(16 / 33)$ survivors in the AKI group who did not receive dialysis, the median renal recovery time was three days (IQR, $2-4$ days; $n=14$ ). Of $10 / 107$ patients who developed AKI after admission, 30\% (3/10) received hemodialysis and 40\% (4/10) died, compared to an overall mortality in the severe malaria cohort of $33 \%(35 / 107)$ (Table 3). The mortality rate among all patients admitted with or developing AKI but not receiving dialysis was nearly double $(20 / 40 ; 50 \%)$ that of patients to those who did (9/32; 28\%)(OR 2.6, 95\% CI, 0.9-7.8; $P=0.09)$. Those with AKI on enrolment had more severe disease, as

Table 1 Baseline demographics and clinical characteristics of patients with severe falciparum malaria by AKI status at enrolment

\begin{tabular}{|c|c|c|c|c|}
\hline Variable & $\begin{array}{l}\text { Total } \\
(n=107)\end{array}$ & $\begin{array}{l}\text { No AKI } \\
(n=45)\end{array}$ & $\begin{array}{l}\text { AKI } \\
(n=62)\end{array}$ & $P$ \\
\hline \multicolumn{5}{|l|}{ Demographics } \\
\hline Age (years) & $30(22-40)$ & $30(25-45)$ & $27(18-40)$ & 0.104 \\
\hline Males $(\%)^{c}$ & $75(70)$ & $35(78)$ & $40(65)$ & 0.200 \\
\hline Fever prior to admission (days) & $7(6-9)$ & $7(6-8)$ & $7(6-9)$ & 0.311 \\
\hline History of black or red urine $e^{c}$ & $20(20)$ & $5(11)$ & $15(26)$ & 0.100 \\
\hline Vomiting and diarrhea (days) & $5(1-6)$ & $2.5(1-5)$ & $5(3-9)$ & 0.045 \\
\hline \multicolumn{5}{|l|}{ Comorbidities } \\
\hline Hypertension $^{c}$ & $5(5)$ & $2(4)$ & $3(5)$ & 1.000 \\
\hline Cardiovascular disease $^{c}$ & $6(6)$ & $2(4)$ & $4(6)$ & 1.000 \\
\hline Type 2 diabetes ${ }^{c}$ & $4(4)$ & $3(7)$ & $1(2)$ & 0.307 \\
\hline \multicolumn{5}{|l|}{ Enrolment clinical parameters } \\
\hline Glasgow Coma Score (max 15) & $9(8-14)$ & $10(9-14)$ & $9(7-14)$ & 0.354 \\
\hline Systolic blood pressure (mmHg) & $110(100-120)$ & $114(102-120)$ & 107 (99-120) & 0.155 \\
\hline Mean arterial pressure $(\mathrm{mmHg})$ & $82(72-90)$ & $81(74-89)$ & $82(71-94)$ & 0.550 \\
\hline Pulse rate (breaths/min) & $115(96-132)$ & $109(93-131)$ & $116(97-132)$ & 0.666 \\
\hline Respiratory rate (breaths/min) & $34(28-42)$ & $32(28-37)$ & $36(28-44)$ & 0.370 \\
\hline Hemoglobinuria on enrolment ${ }^{\# c}$ & $18(17)$ & $4(9)$ & $14(24)$ & 0.124 \\
\hline Number of severity criteria & $2(1-3)$ & $1(1-2)$ & $2(2-4)$ & $<0.001$ \\
\hline \multicolumn{5}{|l|}{ AKI stage at enrolment } \\
\hline Stage $1\left(\geq 1.5 \times{\text { baseline })^{c}}^{c}\right.$ & $16(26)$ & - & $16(26)$ & - \\
\hline Stage $2\left(\geq 2.0-2.9 \times{\text { baseline })^{c}}^{c}\right.$ & $16(26)$ & - & $16(26)$ & - \\
\hline Stage $3(\geq 3.0 \times \text { baseline or } \geq 4 \mathrm{mg} / \mathrm{dl})^{c}$ & $30(48)$ & - & $30(48)$ & - \\
\hline
\end{tabular}

All values are median (IQR) unless otherwise specified: ${ }^{C}$ number (\%). $P<0.05$ using student t-test or Mann-Whitney U. \# hemoglobinuria defined as red, black or dark brown urine on exam with 3/4+ hemoglobin on urine dipstick. Abbreviations: $A K I$ acute kidney injury 
Table 2 Baseline admission laboratory parameters of patients with severe falciparum malaria by AKI status at enrolment

\begin{tabular}{|c|c|c|c|c|c|c|c|}
\hline \multirow[t]{2}{*}{ Variable } & \multirow{2}{*}{$\begin{array}{l}\text { Total } \\
(n=107)\end{array}$} & \multicolumn{2}{|c|}{ No AKI } & \multicolumn{2}{|l|}{$\mathrm{AKI}$} & \multirow{2}{*}{$\begin{array}{l}P \\
\mathrm{n}\end{array}$} & \\
\hline & & $n$ & $(n=45)$ & $n$ & $(n=62)$ & & \\
\hline Hemoglobin (mg/dl) & $9.1(7.2-11.0)$ & 107 & $10.6(8.0-11.3)$ & 45 & $8.5(7.1-10.3)$ & 62 & 0.017 \\
\hline Cell-free hemoglobin $(\mu \mathrm{M})^{\mathrm{b}}$ & $7.0(5.5-8.7)$ & 105 & $5.1(4.0-6.6)$ & 45 & $8.8(6.2-12.3)$ & 60 & 0.018 \\
\hline White blood cells $\left(\times 10^{3} / \mu l\right)$ & $9.4(6.7-12.8)$ & 102 & $9.2(6.4-11.7)$ & 44 & $9.6(7.0-16.6)$ & 58 & 0.339 \\
\hline Platelets $\left(\times 10^{3} / \mu \mathrm{l}\right)$ & $30(19-46)$ & 100 & $35(23-53)$ & 44 & $27(18-42)$ & 56 & 0.075 \\
\hline Total bilirubin (mg/dl) & $2.0(1.0-5.3)$ & 106 & $1.5(0.9-3.2)$ & 45 & $2.5(1.3-10.7)$ & 61 & 0.005 \\
\hline Indirect bilirubin (mg/dl) & $0.8(0.3-1.9)$ & 106 & $0.4(0.2-1.3)$ & 45 & $1.3(0.4-2.7)$ & 61 & 0.013 \\
\hline Lactate dehydrogenase (U/I) & $635(455-886)$ & 107 & $541(403-643)$ & 45 & $766(566-1027)$ & 62 & $<0.001$ \\
\hline Creatinine (mg/dl) & $1.4(1.1-3.3)$ & 107 & $1.2(1.0-1.3)$ & 45 & $3.0(1.6-4.4)$ & 62 & $<0.001$ \\
\hline Blood urea nitrogen (mg/dl) & $43(26-75)$ & 107 & $26(18-37)$ & 45 & $66(44-104)$ & 62 & $<0.001$ \\
\hline Potassium (mmol/l) & $4.4(3.9-5.2)$ & 106 & $4.1(3.8-4.6)$ & 45 & $4.7(4.1-5.5)$ & 61 & $<0.001$ \\
\hline Base excess (mmol/l) & $-8(-11$ to -4$)$ & 107 & $-5(-7$ to -2$)$ & 45 & $-10(-13$ to -7$)$ & 62 & $<0.001$ \\
\hline Bicarbonate (mmol/l) & $17.3(14.1-20.2)$ & 107 & $19.2(17.0-21.9)$ & 45 & $15.9(13.3-18.6)$ & 62 & $<0.001$ \\
\hline Lactate (mmol/l) & $3.85(2.49-6.28)$ & 107 & $3.85(2.58-5.61)$ & 45 & $3.89(2.30-6.68)$ & 62 & 0.852 \\
\hline Parasitemia $(\text { parasites } / \mu l)^{b}$ & $53,529(34586-82,846)$ & 107 & $61,253(34094-110,048)$ & 45 & $48,540(25716-91,619)$ & 62 & 0.605 \\
\hline PfHRP2 (ng/ml) & $2584(1341-7194)$ & 101 & $1743.8(1090-3159)$ & 45 & 3996 (1737-12,382) & 56 & $<0.001$ \\
\hline \multicolumn{8}{|l|}{ Urinary indices } \\
\hline Urine protein:creatinine & $0.81(0.42-1.11)$ & 81 & $0.81(0.52-1.15)$ & 34 & $0.81(0.41-1.11)$ & 47 & 0.867 \\
\hline Urine albumin:creatinine & $9.52(5.49-19.12)$ & 65 & $9.52(5.69-19.12)$ & 25 & $9.76(5.19-19.49)$ & 40 & 0.861 \\
\hline $\mathrm{pH}$ & $5(5-6)$ & 98 & $6(5-6)$ & 42 & $5(5-6)$ & 56 & $<0.001$ \\
\hline $\mathrm{FeNa}(\%)^{\mathrm{C}}$ & $0.67(0.37-1.35)$ & 94 & $0.57(0.22-0.91)$ & 40 & $0.94(0.47-2.44)$ & 54 & 0.012 \\
\hline \multicolumn{8}{|l|}{ Oxidative stress markers } \\
\hline Plasma $F_{2}-$ IsoPs $(\mathrm{pg} / \mathrm{ml})^{\mathrm{b}}$ & $41.1(34.8-48.5)$ & 64 & $27.8(23.7-32.7)$ & 29 & $56.7(45.3-70.9)$ & 35 & $<0.001$ \\
\hline Plasma IsoFs $(\mathrm{pg} / \mathrm{ml})^{\mathrm{b}}$ & $70.6(56.2-88.7)$ & 64 & $41.7(30.2-57.6)$ & 29 & $109.2(85.1-140.1)$ & 35 & $<0.001$ \\
\hline
\end{tabular}

All values are median (IQR) unless otherwise specified: b ${ }^{b}$ geometric mean $(95 \% \mathrm{Cl}),{ }^{c}$ number (\%). $P<0.05$ using student t-test, Mann U Whitney or Fischer's exact tests; significant in bold. Abbreviations: AKI acute kidney injury, PfHRP2 P falciparum histidine rich protein 2, FeNa fractional excretion of sodium, $F_{2}$-IsoPs plasma $\mathrm{F}_{2}$-isoprostanes, IsoFs plasma isofurans

defined by a higher median number of WHO severity criteria $(P<0.001)$. No patient reported a history of kidney disease and there was no difference in comorbidities (hypertension, diabetes and cardiovascular disease) between groups (Table 1).

\section{Cell-free hemoglobin and oxidative stress}

Plasma CFH concentrations were significantly higher in patients with severe malaria $(7.0 \mu \mathrm{M} ; 95 \% \mathrm{CI}$, 5.5-8.7 $\mu \mathrm{M} ; \mathrm{n}=105)$ compared to those with uncomplicated malaria $(4.3 \mu \mathrm{M} ; 95 \% \mathrm{CI}, 3.5-5.2 \mu \mathrm{M}$; $(\mathrm{n}=80)$ Additional file

Table 3 Outcomes by AKI status on enrolment

\begin{tabular}{|c|c|c|c|c|}
\hline \multirow[t]{2}{*}{ Outcome } & Total & No AKI & AKI & \multirow[t]{2}{*}{$P$} \\
\hline & $(n=107)$ & $(n=45)$ & $(n=62)$ & \\
\hline \multicolumn{5}{|l|}{ AKI stage during admissiont } \\
\hline Stage $1(\geq 1.5 \times \text { baseline })^{c}$ & $11(12)$ & $4(10)$ & $7(15)$ & 0.458 \\
\hline Stage $2(\geq 2.0-2.9 \times \text { baseline })^{c}$ & $11(12)$ & $1(2)$ & $10(21)$ & 0.021 \\
\hline Stage $3(\geq 3.0 \times \text { baseline or } \geq 4 \mathrm{mg} / \mathrm{dl})^{c}$ & $36(40)$ & $5(12)$ & $31(65)$ & $<0.001$ \\
\hline Received RRT (\%) ${ }^{c}$ & $32(30)$ & $3(7)$ & $29(47)$ & $<0.001$ \\
\hline Length of hospital stay (days) & $7.6(5.6-12.9)$ & $5.9(4.9-8.3)$ & $10.6(6.6-18.0)$ & $<0.001$ \\
\hline Death $(\%)^{c}$ & $35(33)$ & $10(22)$ & $25(40)$ & 0.061 \\
\hline Study hours to death $(\%)^{c}$ & $22.5(12.5-51.0)$ & $40.1(19.0-115.0)$ & $21.0(10.0-38.0)$ & 0.074 \\
\hline
\end{tabular}

All values are median (IQR) unless otherwise specified: ' number (\%). $P<0.05$ using Mann $U$ Whitney or Fischer's exact test; significant in bold. + Highest KDIGO stage during admission in those that survived more than $24 \mathrm{~h}$. Abbreviations: AKI = acute kidney injury; RRT = renal replacement therapy 
1: Figure S1; $P=0.002)$ or sepsis $(2.5 \mu \mathrm{M} ; 95 \% \mathrm{CI}, 1.4-$ $4.3 \mu \mathrm{M} ; n=28 ; P<0.001$ ). Plasma $\mathrm{F}_{2}$-IsoPs and IsoFs were also higher in patients with severe malaria compared to uncomplicated malaria (Additional file 1: Figure S1; $F_{2}$-IsoPs, $P<0.001$; IsoFs, $\left.P=0.005\right)$. Among those with severe malaria, plasma $\mathrm{CFH}, \mathrm{F}_{2}$-IsoPs and IsoFs correlated with other measures of hemolysis, including LDH, total bilirubin, and indirect bilirubin. PfHRP2, [26] but not peripheral blood parasitemia, correlated positively with CFH $\left(r_{s}=0.55, P<0.001\right.$; $r_{s}=0.18, P=0.08$ ) (Fig. 1A). Similarly, $F_{2}$-IsoPs and IsoFs correlated with PfHRP2 $\left(r_{s}=0.34, P=0.008\right.$; $\left.r_{s}=0.31, P=0.017\right)$ but neither correlated with parasitemia $\left(r_{s}=0.02, P=0.89 ; r_{s}=-0.03, P=0.98\right)$. $\mathrm{CFH}$ weakly correlated with plasma $\mathrm{F}_{2}$-IsoPs $\left(r_{s}=0.30\right.$, $P=0.018$ ), as a measure of oxidative stress (Fig. 1B); but the correlation with IsoFs was not significant $\left(r_{s}=0.16, P=0.22\right)$. Both plasma $\mathrm{F}_{2}$-IsoPs and IsoFs were inversely associated with $\mathrm{pH}\left(r_{s}=-0.51\right.$, $P<0.001 ; r_{s}=-0.49 ; P<0.001$ ) (Fig. 1C, D) and positively correlated with base deficit $\left(r_{s}=0.59\right.$, $\left.P<0.001 ; r_{s}=0.63 ; P<0.001\right)$. In a multivariable regression model adjusting for disease severity, $\mathrm{CFH}$ and decreasing $\mathrm{pH}$ (acidosis) were positive predictors of $(\log ) \mathrm{F}_{2}$-IsoPs ( $\beta$ coefficient $0.13 ; 95 \% \mathrm{CI}, 0.02$ to $0.25 ; \quad P=0.026 ;-2.94 ; 95 \% \mathrm{CI},-4.24$ to -1.64 ; $P<0.001$, respectively).

Cell-free hemoglobin, oxidative stress, and renal function In severe malaria, $\mathrm{CFH}$ was higher in patients with AKI compared to those without AKI $(P=0.018)$ (Fig. 2; Table 2). CFH in patients with severe malariaassociated AKI was also higher compared to patients with sepsis-related AKI $(0.8 \mu \mathrm{M} ; 95 \% \mathrm{CI}, 0.1-3.9 \mu \mathrm{M}$; $n=4 ; P=0.001)$. Patients with hemoglobinuria at enrolment had significantly higher $\mathrm{CFH}$ (geometric mean: $15.6 \mu \mathrm{M}$; 95\% CI, 6.9-35.6 $\mu \mathrm{M} ; n=18$ ), PfHRP2 (median: 10,411 ng/ml; IQR, 2909-14,504 ng/ml; $n=18$ ), and serum creatinine (median: $2.9 \mathrm{mg} / \mathrm{dl}$; IQR, $1.3-4.7 \mathrm{mg} / \mathrm{dl} ; n=18$ ) compared to those without hemoglobinuria (CFH: 5.4 $\mu \mathrm{M}$; 95\% CI, 4.3-6.7 $\mu \mathrm{M}$; $n=74 ; P<0.001$; PfHRP2: $2146 \mathrm{ng} / \mathrm{ml} ; \mathrm{IQR}, 1266-$ $4216 \mathrm{ng} / \mathrm{ml} ; n=73 ; P=0.001$; creatinine: $1.4 \mathrm{mg} / \mathrm{dl}$; IQR, $1.1-2.7 \mathrm{mg} / \mathrm{dl} ; n=76 ; P=0.040)$. In severe
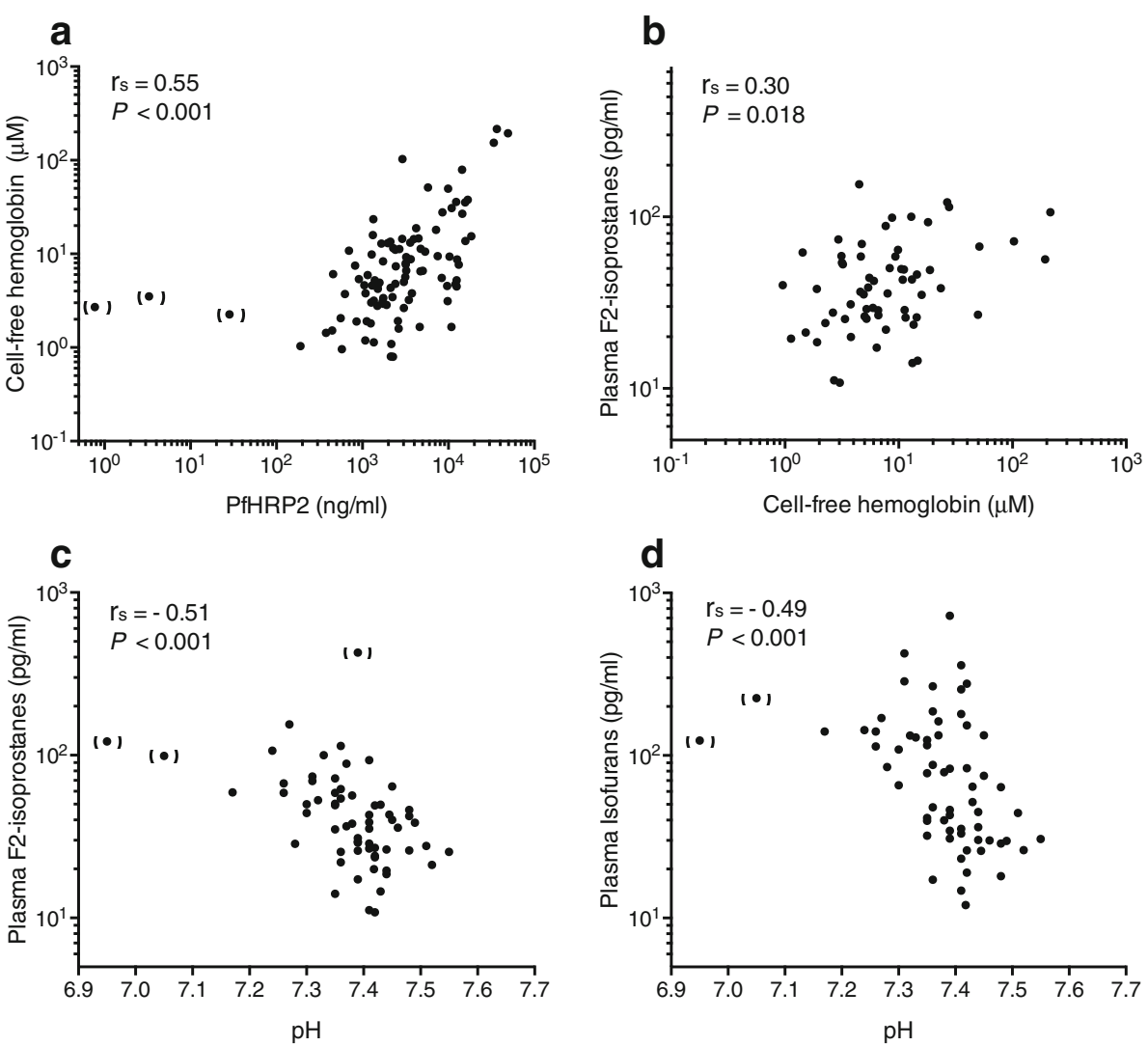

Fig. 1 Correlation of oxidative stress markers in severe malaria. a Parasite burden, as measured by PfHRP2 concentration, positively correlated with plasma cell-free hemoglobin concentration $(n=96)$, b cell-free hemoglobin positively correlated with plasma $F_{2}$-isoprostanes $(n=62)$. Acidemia (venous $\mathrm{pH}$ ) was inversely correlated with (c) plasma $\mathrm{F}_{2}$-isoprostanes, and $\mathbf{d}$ plasma isofurans. All values are from enrolment assessments. Abbreviations: PfHRP2, Plasmodium falciparum histidine rich protein 2; $r_{5}$, Spearman's correlation coefficient 


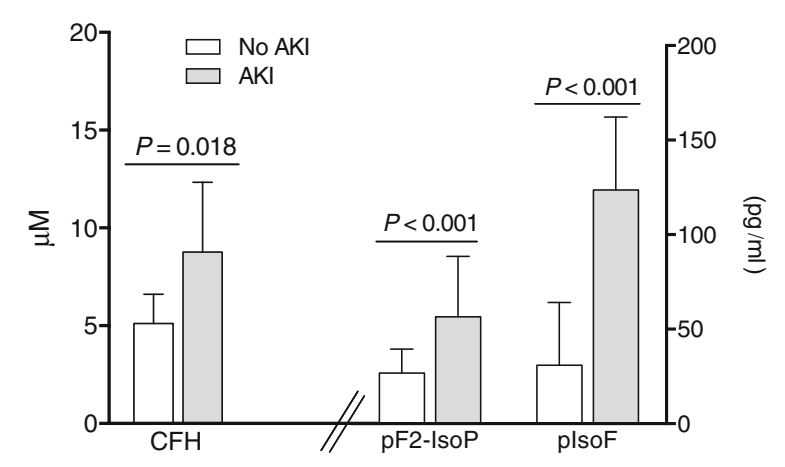

Fig. 2 Cell-free hemoglobin, and oxidative stress measures at enrolment in patients with severe malaria by AKI status. Plasma cell-free hemoglobin $(n=105), F_{2}$-isoprostanes $(n=64)$ and isofurans ( $n=64)$ were significantly more elevated on enrolment in those with acute kidney injury. Geometric mean and 95\% Cl are shown. Abbreviations: $\mathrm{AKl}$, acute kidney injury; $\mathrm{CFH}$, cell-free hemoglobin; $\mathrm{pF}_{2}$-IsoP, plasma $\mathrm{F}_{2}$-isoprostanes; plsoF, plasma isofurans

malaria, plasma $F_{2}$-IsoPs and IsoFs were significantly higher in patients with AKI compared to those without (both $P<0.001$ ) (Fig. 2; Table 2). Enrolment plasma $\mathrm{F}_{2}$-IsoPs and IsoFs strongly correlated with enrolment serum creatinine $\left(r_{s}=0.71, P<0.001 ; r_{s}=0.71\right.$, $P<0.001$ ) (Fig. 3A, B) and with the fractional excretion of sodium $\left(r_{s}=0.49, P<0.001 ; r_{s}=0.37\right.$, $P=0.006)$. The 24-h urine $\mathrm{F}_{2}$-IsoP excretion concentration was lower in the AKI group (median: $0.9 \mathrm{ng}$; IQR, 0.6-2.4 ng; $n=9$ ) compared to those without AKI (2.9 ng; IQR, $1.1-4.0 \mathrm{ng} ; n=11 ; P=0.037$ ). However, the 24-h urine IsoF excretion concentration was similar between groups (AKI median: $9.7 \mathrm{ng}$; IQR, 8.1-15.2 ng; $n$ = 9; No AKI: 9.2 ng; IQR, 4.3-53.2 ng; $n=12 ; P=0.72$ ).

In patients without AKI on enrolment but subsequently developing AKI, initial plasma IsoFs were higher compared to those who did not develop AKI (geometric mean: $81.6 \mathrm{pg} / \mathrm{ml} ; 95 \% \mathrm{CI}, 28.6-232.9 \mathrm{pg} / \mathrm{ml}$ versus $35.0 \mathrm{pg} / \mathrm{ml} ; 95 \% \mathrm{CI}, 25.6-48.0 \mathrm{pg} / \mathrm{ml} ; P=0.027)$. Furthermore, in this group (excluding $3 / 10$ patients who received hemodialysis) peak plasma concentrations of $\mathrm{F}_{2}$-IsoPs and IsoFs at $24 \mathrm{~h}$ were higher than in patients not developing AKI ( $\mathrm{F}_{2}$-IsoPs: $43.5 \mathrm{pg} / \mathrm{ml}$; 95\% CI 26.4$71.8 \mathrm{pg} / \mathrm{ml}$; versus $24.8 \mathrm{pg} / \mathrm{ml}$; $95 \%$ CI $19.1-32.1 \mathrm{pg} / \mathrm{ml}$; $P=0.020$; IsoF: geometric mean $220.0 \mathrm{pg} / \mathrm{ml} ; 95 \% \mathrm{CI}$, $52.9-914.8 \mathrm{pg} / \mathrm{ml}$ versus $48.0 \mathrm{pg} / \mathrm{ml} ; 95 \% \mathrm{CI}, 32.8-$ $70.2 \mathrm{pg} / \mathrm{ml} ; P=0.003)$. The peak creatinine in the former group was reached at $48 \mathrm{~h}$ (median: $4.5 \mathrm{mg} / \mathrm{dl}$; IQR, $1.1-4.6 \mathrm{mg} / \mathrm{dl})$.

Plasma $F_{2}$-IsoPs and IsoFs on enrolment, prior to hemodialysis, were higher among those who received hemodialysis ( $\mathrm{F}_{2}$-IsoPs: $58 \mathrm{pg} / \mathrm{ml}$; $95 \% \mathrm{CI}, 46-72 \mathrm{pg} / \mathrm{ml}$; $n=19$; versus $36 \mathrm{pg} / \mathrm{ml} ; 95 \% \mathrm{CI}, 29-44 \mathrm{pg} / \mathrm{ml} ; n=45$; $P=0.006$; IsoFs: $131 \mathrm{pg} / \mathrm{ml} ; 95 \% \mathrm{CI}, 99-173 \mathrm{pg} / \mathrm{ml}$; $n=19$; versus $54 \mathrm{pg} / \mathrm{ml} ; 95 \% \mathrm{CI}, 41-71 \mathrm{pg} / \mathrm{ml} ; n=45$; $P<0.002)$. Plasma CFH at enrolment was not higher in those who received hemodialysis $(\mathrm{CFH}: 9.0 \mu \mathrm{M}$; 95\% CI, 5.2-15.4 $\mu \mathrm{M} ; n=31$; versus $6.2 \mu \mathrm{M} ; 95 \% \mathrm{CI}, 4.9-$ $7.9 \mu \mathrm{M} ; n=74 ; P=0.15$ ).

\section{Red cell deformability, renal function and oxidative stress} RCD at enrolment was lower at shear stresses between 1.69 and $30.00 \mathrm{~Pa}$ in patients with AKI on enrolment compared to those without AKI (Figure 4). Decreased RCD correlated with higher creatinine at all these shear stresses and most strongly at $9.49 \mathrm{~Pa}\left(r_{s}=-0.31\right.$, $P=0.019)$. RCD was also lower at shear stresses between 1.69 and $9.49 \mathrm{~Pa}$ in patients who subsequently required hemodialysis (all $P<0.05$ ). RCD at low shear stress was inversely associated with plasma $F_{2}$-IsoPs (0.3 Pa: $\left.r_{s}=-0.46, P=0.003 ; n=39\right)$ but not with IsoFs (1.69 Pa: $r_{s}=-0.30, P=0.062 ; n=40$ ).
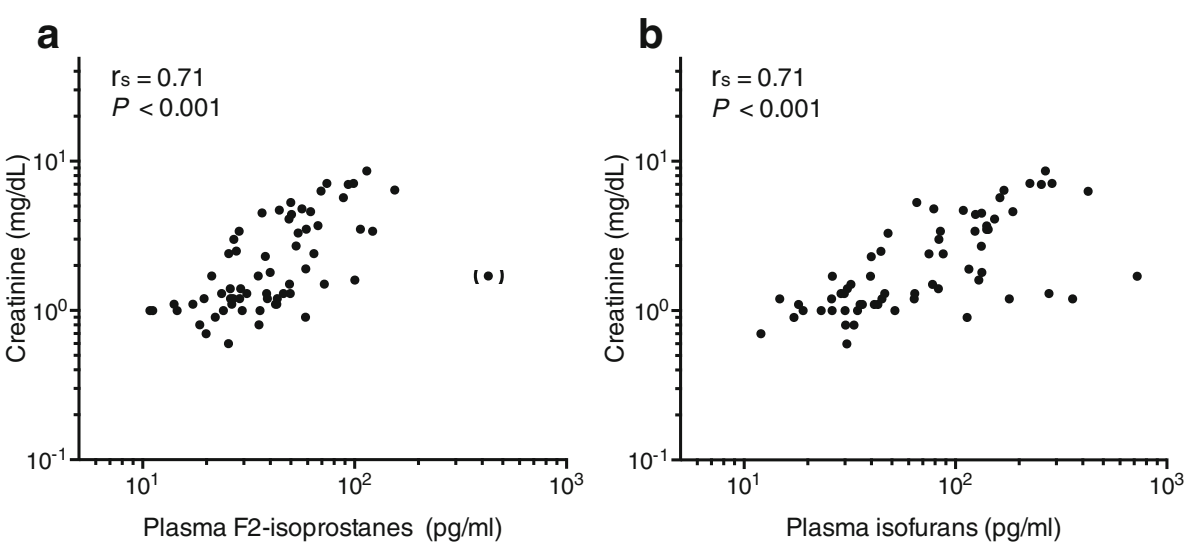

Fig. 3 Correlations with creatinine in patients with severe malaria. Creatinine on enrolment positively correlated with (a) plasma $F_{2}$-isoprostanes $(n=63)$, and (b) plasma isofurans $(n=64)$. Abbreviations: $r_{s}$, Spearman's correlation coefficient 


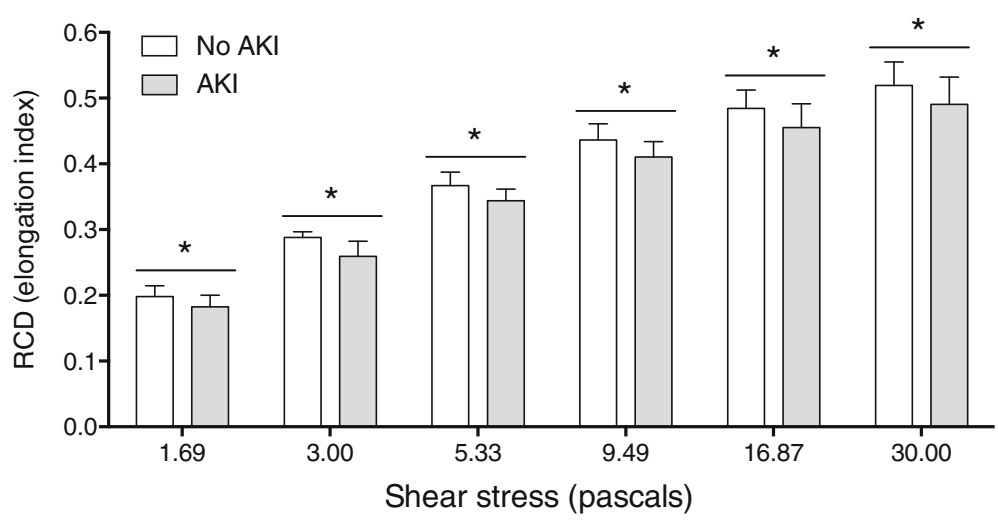

Fig. 4 Red cell deformability at enrolment in patients with severe malaria by AKI status. Red cell deformability at shear stresses from 1.69 to 30.0 Pa were significantly lower in the AKI group $(n=51)$ compared to the no AKI group $(n=33)$. Abbreviations: AKl, acute kidney injury; RCD, red cell deformability. Asterisks represent $P<0.05$ significance

\section{Predictors of renal function}

Since plasma $\mathrm{F}_{2}$-IsoPs and IsoFs were collinear, two different multivariable mixed effects models were considered to assess the associations of each of them with change in serum creatinine over time (Table $4, \mathrm{~F}_{2}$-IsoP model and IsoF model, respectively). There was a significant interaction between $(\log ) \mathrm{CFH}$ and time in both multivariable models, $P=0.010$ and $P=0.012$ for the model that included $(\log ) \mathrm{F}_{2}$-IsoPs and $(\log )$ IsoFs, respectively (Table 4$)$. The effect of enrolment $\mathrm{CFH}$ on creatinine had a lag time. In the model with $\mathrm{F}_{2}$-IsoPs, increased enrolment plasma $\mathrm{F}_{2}$-IsoPs, PfHRP2 and decreased red cell deformability were also independently associated with increasing serum creatinine over the first $72 \mathrm{~h}$ of admission. In the model with IsoFs, increased enrolment plasma IsoFs, and PfHRP2 were independently associated with an increase in serum creatinine over $72 \mathrm{~h}$. In a logistic regression model adjusted for disease severity, plasma $\mathrm{F}_{2}$-IsoPs $(\mathrm{OR}=7.37$, 95\% CI, 1.86-29.23) was independently associated with the need for hemodialysis during admission, but RCD was not (overall model fit $R^{2}=0.20$; Table 5). In the logistic regression model with IsoFs (rather than $\mathrm{F}_{2}$-IsoPs) as independent variable, elevated IsoFs $(\mathrm{OR}=5.5,95 \% \mathrm{CI}$, 1.76-17.24) at enrolment was also independently

Table 4 Association of variables with change in absolute creatinine over $72 \mathrm{~h}$ in patients with severe malaria

\begin{tabular}{|c|c|c|c|c|c|c|}
\hline \multirow[b]{2}{*}{ Variable } & \multicolumn{2}{|l|}{ Univariable analysis } & \multicolumn{2}{|c|}{ Multivariable $F_{2}$-IsoP model } & \multicolumn{2}{|c|}{ Multivariable IsoF model } \\
\hline & $\overline{\beta(95 \% C l)^{a}}$ & $P$ & $\overline{\beta(95 \% C l)^{a}}$ & $P$ & $\overline{\beta(95 \% C l)^{a}}$ & $P$ \\
\hline $\log C F H^{\S}$ & $0.27(-0.19$ to 0.74$)$ & 0.249 & - & - & - & - \\
\hline Log $F_{2}$-IsoP\# & 2.35 (1.27 to 3.43$)$ & $<0.001$ & 3.01 (1.92 to 4.12$)$ & $<0.001$ & - & - \\
\hline Log IsoF & 2.06 (1.46 to 2.65$)$ & $<0.001$ & - & - & 1.83 (1.26 to 2.40$)$ & $<0.001$ \\
\hline LogPfHRP2 & 0.61 (0.25 to 0.98$)$ & 0.001 & 0.70 (0.02 to 1.38$)$ & 0.045 & 0.69 (0.05 to 1.32$)$ & 0.034 \\
\hline RCD at SS $1.69 \mathrm{~Pa}$ & $-1.67(-14.93$ to 11.59$)$ & 0.805 & - & - & - & - \\
\hline RCD at SS $9.49 \mathrm{~Pa}$ & $-6.42(-15.10$ to 2.26$)$ & 0.147 & $-4.26(-7.86$ to -0.67$)$ & 0.020 & $-2.46(-5.92$ to 1.00$)$ & 0.163 \\
\hline Age & $0.01(-0.03$ to 0.05$)$ & 0.624 & - & - & - & - \\
\hline SBP & $0.03(-0.01$ to 0.06$)$ & 0.094 & & & - & - \\
\hline Visit (time) & 0.65 (0.52 to 0.78$)$ & $<0.001$ & - & - & - & - \\
\hline $\log C F H \times \log F_{2}-I s o P$ & $0.31(-0.19$ to 0.86$)$ & 0.221 & - & - & - & - \\
\hline $\log \mathrm{CFH} \times \log$ IsoF & 0.62 (0.27 to 0.98$)$ & 0.001 & - & - & - & - \\
\hline Log $F_{2}-$ IsoP $\times$ visit & 0.85 (0.55 to 1.14$)$ & $<0.001$ & - & - & - & - \\
\hline Log IsoF $\times$ visit & 0.62 (0.47 to 0.76 ) & $<0.001$ & - & - & - & - \\
\hline Log CFH $\times$ visit & $0.10(-0.10$ to 0.21$)$ & 0.076 & 0.21 (0.05 to 0.36 ) & 0.010 & 0.20 (0.04 to 0.36$)$ & 0.012 \\
\hline
\end{tabular}

${ }^{a}$ Regression coefficient $(\beta)$ with $95 \%$ confidence intervals $(\mathrm{Cls})$ showing the estimated decrease in creatinine predicted by a 1-U change in the independent (predictor) variables. Interaction terms improved the model fit. Abbreviations: $C F H$ cell free hemoglobin, $F_{2}-$ IsoP plasma $\mathrm{F}_{2}$-isoprostanes, IsoF plasma isofurans, $C F H \times F_{2}-I s O P$ cell-free hemoglobin and plasma $F_{2}$-isoprostane interaction term, CFH $\times I s O F$ cell-free hemoglobin and plasma isofuran interaction term, PfHRP2 $P$. falciparum histidine rich protein $2, R C D$ red cell deformability at shear stress 1.69 and $9.49 \mathrm{~Pa}$. \# Plasma IsoFs collinear with plasma $\mathrm{F}_{2}-$ IsoPs. P-values in italics denote statistical significance 
Table 5 Association of variables with subsequent hemodialysis requirement in patients with severe malaria

\begin{tabular}{|c|c|c|c|c|c|c|}
\hline \multirow[b]{2}{*}{ Variable } & \multicolumn{2}{|l|}{ Univariable analysis } & \multicolumn{2}{|c|}{ Multivariable $F_{2}$-IsoP model } & \multicolumn{2}{|c|}{ Multivariable IsoF model } \\
\hline & $\mathrm{OR}(95 \% \mathrm{Cl})^{\mathrm{a}}$ & $P$ & $\overline{\mathrm{OR}(95 \% \mathrm{Cl})^{\mathrm{a}}}$ & $P$ & $\overline{\mathrm{OR}}(95 \% \mathrm{Cl})^{\mathrm{a}}$ & $P$ \\
\hline$\overline{\mathrm{CFH}}$ & 1.01 (0.99 to 1.03 ) & 0.063 & - & - & 1.06 (0.98 to 1.14$)$ & 0.156 \\
\hline Log $F_{2}$-IsoP\# & 3.45 (1.30 to 9.16$)$ & 0.013 & 7.37 (1.86 to 29.23 ) & 0.005 & - & - \\
\hline Log IsoF & 3.48 (1.62 to 7.49$)$ & 0.001 & - & - & 5.50 (1.76 to 17.24$)$ & 0.003 \\
\hline LogPfHRP2 & $1.92(1.27$ to 2.91$)$ & 0.002 & - & - & - & - \\
\hline LogRCD at SS $1.69 \mathrm{~Pa}$ & 0.29 (0.08 to 1.09$)$ & 0.066 & - & - & - & - \\
\hline LogRCD at SS 9.49 Pa & 0.10 (0.01 to 0.75$)$ & 0.025 & 0.057 (0.002 to 1.33$)$ & 0.075 & 0.031 (0.001 to 1.44$)$ & 0.076 \\
\hline Age & 1.00 (0.97 to 1.03$)$ & 0.940 & - & - & - & - \\
\hline SBP & 1.02 (0.99 to 1.04$)$ & 0.260 & & & - & - \\
\hline Number of severity criteria & 1.90 (1.38 to 2.62$)$ & $<0.001$ & - & - & - & - \\
\hline GCS & 0.95 (0.85 to 1.07$)$ & 0.393 & - & - & - & - \\
\hline LogLactate & 1.22 (0.64 to 2.30$)$ & 0.548 & - & - & - & - \\
\hline
\end{tabular}

${ }^{\mathrm{a}}$ Odds ratio (OR) with $95 \%$ confidence intervals (Cls) showing the hemodialysis requirement predicted by a 1-U change in the independent (predictor) variables. A backward stepwise multivariable model included all variables in univariable analyses which were removed on the basis of $P \geq 0.05$. Abbreviations: $C F H$ cell free hemoglobin, $F_{2}$-IsoP plasma $F_{2}$-isoprostanes, IsoF plasma isofurans, PfHRP2 $P$. falciparum histidine rich protein $2, R C D$ red cell deformability at shear stress 1.69 and 9.49 Pa, SBP systolic blood pressure, GCS Glasgow Coma Score. \# Plasma IsoFs collinear with plasma $F_{2}$ - IsoPs. P-values in italics denote statistical significance

associated with the subsequent need for hemodialysis, but RCD and CFH were not (overall model fit $R^{2}=0.41$; Table 5).

\section{Cell-free hemoglobin, oxidative stress and survival}

Those who died had higher enrolment plasma $\mathrm{F}_{2}$-IsoPs (geometric mean: $55 \mathrm{pg} / \mathrm{ml}$; 95\%CI, 38-79; $n=21$ ) and IsoFs (geometric mean: $97 \mathrm{pg} / \mathrm{ml}$; 95\%CI, 64-145; $n=21$ ) compared to survivors (F F $_{2}$ IsoPs: $36 \mathrm{pg} / \mathrm{ml}$; 95\%CI, 30-42; $n=43 ; P=0.014 ;$ IsoFs: $61 \mathrm{pg} / \mathrm{ml}$; $95 \% \mathrm{CI}, 46-80 ; n=43 ; P=0.054)$. There was no difference in $\mathrm{CFH}$ between those who died and survived $(P=0.143)$.

\section{Discussion}

In this study, plasma cell-free hemoglobin and oxidative stress markers ( $\mathrm{F}_{2}$-IsoPs and IsoFs) were strongly associated with the presence of AKI on admission, and oxidative stress markers predicted subsequent creatinine elevation and hemodialysis requirement during admission in adult patients with severe falciparum malaria.

Earlier studies have shown that urine $\mathrm{F}_{2}$-IsoPs and other urine and plasma oxidative stress markers are elevated in severe malaria compared to uncomplicated malaria [18-21]. CFH-mediated oxidative stress has been shown to contribute to AKI in diseases and medical procedures inducing hemolysis [15-17, 35, 36]. In malaria, intravascular hemolysis involves both parasitized and non-parasitized red blood cells [2]. In the current study, plasma CFH was associated with higher parasite burden, as measured by PfHRP2, consistent with a previous study [1]. This suggests that the hemoglobin released at schizont rupture, the end of the 48-h intra- erythrocytic lifecycle, contributes to plasma CFH concentration.

Redox cycling of hemoglobin forms a radical species that can initiate lipid peroxidation of arachidonic acid to generate $\mathrm{F}_{2}$-IsoPs and IsoFs $[11,12,37]$. In this study, $\mathrm{CFH}$ concentrations correlated with increased levels of plasma $F_{2}$-IsoPs, a marker of oxidative stress. Several factors influence the generation of lipid peroxidation markers. The oxidative capacity of plasma $\mathrm{CFH}$ is largely dependent on its redox state and the fate of the heme moiety. Heme is water-insoluble; it binds to hemopexin, albumin, lipoproteins, and cell membranes [38], and its oxidative capacity differs considerably between these fractions. In addition, other sources of free radicals, such as those created during phagocytic oxidative burst, may initiate lipid peroxidation to generate plasma $\mathrm{F}_{2}$-IsoPs and IsoFs. Indeed, renal histopathology of AKI in severe malaria shows accumulation of host monocytes (in addition to parasitized red blood cells) in the renal microvasculature [39]. Heme-containing myoglobin concentrations are also increased in severe malaria, although to a much lesser extent than CFH [1]. The oxidative capacity of myoglobin increases at low $\mathrm{pH}$ because of increased pseudoperoxidase activity [10]. This could also apply to $\mathrm{CFH}$, given that both plasma $\mathrm{F}_{2}$-IsoPs and IsoFs were associated with reduced venous blood $\mathrm{pH}$ in the current study.

$\mathrm{F}_{2}$-IsoPs are potent renal vasoconstrictors, which reduce renal blood flow and GFR [12]. In this study, concentrations of plasma $\mathrm{CFH}, \mathrm{F}_{2}$-IsoPs, and IsoFs at enrolment were all higher in patients with $\mathrm{AKI}$, whereas $\mathrm{F}_{2}$-IsoPs and IsoFs correlated with the fractional excretion of sodium (an indirect measure of renal tubular 
injury) and were independently associated with increased creatinine over $72 \mathrm{~h}$ and the subsequent requirement for hemodialysis. A common problem of AKI biomarkers is that their concentrations can be increased as a result of renal dysfunction, rather than be a cause of it. Indeed, the lower 24-h urine $\mathrm{F}_{2}$-IsoP excretion concentrations in patients with established AKI suggest that hemoglobin dimers and/or $\mathrm{F}_{2}$-IsoPs are not filtered well at low creatinine clearances. However, the group of patients without AKI on enrolment that subsequently developed AKI also showed elevated enrolment plasma IsoF concentrations. This was then followed by concomitant increases in $24 \mathrm{~h}$ plasma $\mathrm{F}_{2}$-IsoPs and IsoF with a subsequent peak in serum creatinine at $48 \mathrm{~h}$. This time course suggests a role of plasma $\mathrm{F}_{2}$-IsoPs and IsoFs in the pathogenesis of AKI. The delayed effect of $\mathrm{CFH}-$ mediated oxidative stress on renal function is also suggested by the multivariable mixed effects model, in which time modified the effect of enrolment CFH on creatinine, where the slope of change in creatinine increased with time.

Plasma $\mathrm{F}_{2}$-IsoPs were also associated with reduced $\mathrm{RBC}$ deformability. Red cell rigidity has been well-described in severe malaria [27]. It is thought to be caused by oxidative damage to RBC membranes, and contribute to microvascular flow obstruction [27, 28]. RBC membranes are rich in arachidonic acid. Heme-mediated lipid peroxidation of RBC membranes could be an important cause of reduced $R C D$, and a significant source of plasma $F_{2}$-IsoPs and IsoFs in severe malaria. RCD at low shear stresses is mainly determined by membrane properties, whereas at high shear stresses surface-to-volume relationships become more important $[40,41]$. In this study, the correlation between plasma $\mathrm{F}_{2}$-IsoPs and decreased RCD was strongest at low shear stress, suggesting RBC membrane damage. In the current study, RCD was lower in patients with AKI, inversely correlated with serum creatinine, and independently associated with an increase in serum creatinine over 72 h. Reduced RCD could further compromise renal medullary perfusion concomitant with $\mathrm{F}_{2}$-IsoP- and IsoF-induced vasoconstriction and obstructing microvascular sequestered parasitized red blood cells, all contributing to renal tubular damage [39].

This study has some limitations. The sample size of this detailed study was relatively small. Additional biomarkers of AKI were not assessed. However, both changes in serum creatinine and hemodialysis are well established indicators of kidney dysfunction and injury. Living or post-mortem renal biopsy as a standard to evaluate the underlying kidney pathology was not ethical or feasible in this study. Quantification of plasma and urine hemoglobin oxidation states was not performed. However, studies have shown that methemoglobin is the predominant form in patients with malaria and blackwater fever [42]. Myoglobin as an alternative source of heme-mediated oxidative damage was not assessed, but other studies have shown a 100 -fold less increase in plasma myoglobin compared to $\mathrm{CFH}$ in patients with severe malaria [1].

\section{Conclusions}

In summary, the findings of this study suggest that $\mathrm{CFH}$ and systemic $\mathrm{F}_{2}$-IsoPs and IsoFs contribute to the pathogenesis of AKI in severe malaria and these markers of oxidative stress are associated with need for hemodialysis and in-hospital mortality. $\mathrm{CFH}$ can have a direct oxidative damaging effect on renal tubules. In addition, $\mathrm{CFH}$ induced lipid peroxidation may have a dual effect in the mechanism of AKI in malaria. Firstly, the lipid peroxidation metabolites, plasma $\mathrm{F}_{2}$-IsoPs, may cause direct renal vasoconstriction. Secondly, lipid peroxidation of $\mathrm{RBC}$ membranes may result in reduced RCD, which in turn could exacerbate ischemia in the renal medulla.

Therapies targeted at reducing hemoprotein-mediated oxidative stress have been shown to improve renal function and mortality $[9,17,35]$. Treatments that reduce $\mathrm{CFH}$ levels or $\mathrm{CFH}$-mediated oxidative stress, such as haptoglobin or paracetamol respectively, may have potential as an adjunctive therapy to improve kidney function and survival in severe malaria.

\section{Additional file}

\begin{abstract}
Additional file 1: Figure S1. Supplement to Plewes K, Kingston HWF, Ghose A, et al. Cell-free hemoglobin mediated oxidative stress is associated with acute kidney injury and renal replacement therapy in severe falciparum malaria: an observational study. Cell-free hemoglobin, and oxidative stress measures at enrolment stratified by malaria severity. Plasma cell-free hemoglobin $(n=185), F_{2}$-isoprostanes $(n=82)$ and isofurans ( $n=82$ ) were significantly more elevated on enrolment in those with severe malaria compared to uncomplicated malaria. Geometric mean and 95\% Cls shown. Abbreviations: AKl, acute kidney injury; CFH, cell-free hemoglobin; $\mathrm{pF}_{2}$-IsoP, plasma $\mathrm{F}_{2}$-isoprostanes; plsoF, plasma isofurans. (DOCX $1707 \mathrm{~kb}$ )
\end{abstract}

\section{Abbreviations}

AKl: acute kidney injury; CFH: cell-free hemoglobin; F2-IsoPs: F2-isoprostanes; GFR: glomerular filtration rate; IsoF: Isofurans; KDIGO: Kidney Diseases Improving Global Outcomes; PfHRP2: Plasmodium falciparum histidine rich protein 2; RBC: red blood cell; RCD: red cell deformability; RRT: renal replacement therapy

\section{Acknowledgments}

We thank the patients, relatives, research assistants, attending physicians, and support staff at Chittagong Medical College Hospital for their assistance and collaboration with the Mahidol-Oxford Tropical Medicine Research Unit. Benjamas Intharabut, Ketsanee Srinamon, Md Safiqul Mostafa Choudury, Sanjib Kanti Paul, and Sumon Sharma for their instrumental assistance. We would also like to acknowledge Bill Zachert for performing quantification of $\mathrm{F}_{2}$-isoprostanes and isofurans.

\section{Funding}

This work was supported by the Wellcome Trust of Great Britain (grant number 089275/Z/09/Z); the Australian National Health and Medical Research Council (grant number 605807, and Fellowships to NMA and TWY), and the National Institutes of Health (grant GM15431 to LJR). KP was supported by the Infectious Diseases Society of America, Education and Research Foundation and the 
National Foundation for Infectious Diseases, Young Investigator recipient of the Merle A. Sande/Pfizer Fellowship in International Infectious Diseases; and the Clinician Investigator Program at the University of British Columbia, Canada.

\section{Availability of data and materials}

The datasets generated and analyzed during the current study are not publicly available. The Mahidol Oxford Tropical Medicine Research Unit has a Data Access Committee that reviews all data requests on a case by case basis. MORU is committed to ensuring that data sharing is planned for at the inception of a study: including during negotiations with funders and collaborating sites, during evaluation of compliance with local and international ethics and regulatory requirements, and during the design and conduct of consent processes. Queries and applications for datasets should be directed to the corresponding author who will discuss with the MORU Data Access committee. For further information please refer to the MORU Data Sharing Policy (http://www.tropmedres.ac/data-sharing-policy).

\section{Authors' contributions}

LR and AMD conceived of the study. KP contributed to study design, patient enrollment, acquisition of patient samples, performed the statistical analysis and wrote the manuscript. HWK, RJM, MTH, SJL, HI, TWY, PC, and KS contributed to the study design, patient enrolment, sample collection and manuscript revision. AG, MMUH, MSH, SA and MAH contributed to study design, clinical care of the patients and manuscript revision. KAP performed the cell-free hemoglobin measurements, and provided interpretation of the data and revision of the manuscript. SJL, MM and AMD advised and assisted with statistical analysis, and critically revised the manuscript for intellectual content. LJR provided the $F_{2}-I s O P$ and IsoF quantification. MAF, GDHT, TWY, NMA, NPJD, NJW, $\sqcup R$, and AMD provided interpretation of the data and critical manuscript revisions. All authors read and approved of the final manuscript, and agreed to be accountable for all aspects of the work.

\section{Competing interests}

All authors declare that they have no competing interests.

\section{Consent for publication}

Informed written consent for publication was obtained from each patient or legally acceptable representative.

\section{Ethics approval and consent to participate}

Informed written consent to participate was obtained from each patient or legally acceptable representative. All procedures were in accordance with the ethical standards of Declaration of Helsinki 2008. Ethical approval was obtained from Chittagong Medical College Ethical Review Committee, and Oxford Tropical Research Ethics Committee (reference 21-11).

\section{Publisher's Note}

Springer nature remains neutral with regard jurisdictional claims in published maps and institutional affiliations.

\footnotetext{
Author details

${ }^{1}$ Mahidol Oxford Tropical Medicine Research Unit, Faculty of Tropical Medicine, Mahidol University, Bangkok, Thailand. ${ }^{2}$ Centre for Tropical Medicine and Global Health, Nuffield Department of Medicine, University of Oxford, Oxford, UK. ${ }^{3}$ Department of Medicine and Vancouver Coastal Health, University of British Columbia Clinical Investigator program, Vancouver, Canada. ${ }^{4}$ Global Health Division, Menzies School of Health Research and Charles Darwin University, Darwin, Northern Territory, Australia. ${ }^{5}$ Department of Medicine, Chittagong Medical College and Hospital, Chittagong, Bangladesh. ${ }^{6}$ Department of Nephrology, Chittagong Medical College and Hospital, Chittagong, Bangladesh. ${ }^{7}$ Department of Anesthesiology, Chittagong Medical College and Hospital, Chittagong, Bangladesh. ${ }^{8}$ Lee Kong Chian School of Medicine, Nanyang Technological University, Singapore, Singapore. 'Dev Care Foundation, Dhaka, Bangladesh. ${ }^{10}$ Department of Pharmacology, Vanderbilt University, Nashville, TN, USA.
}

Received: 19 October 2016 Accepted: 30 March 2017 Published online: 27 April 2017

\section{References}

1. Yeo TW, Lampah DA, Tjitra E, Gitawati R, Kenangalem E, Piera K, Granger DL, Lopansri BK, Weinberg JB, Price RN, Duffull SB, Celermajer DS, Anstey NM. Relationship of cell-free hemoglobin to impaired endothelial nitric oxide bioavailability and perfusion in severe falciparum malaria. J Infect Dis. 2009; 200:1522-9.

2. Foy $\mathrm{H}$, Kondi A, Rebelo A, Soeiro A. Survival of transfused red cells in blackwater fever circulation and of blackwater red cells in normal circulation (preliminary report). Trans R Soc Trop Med Hyg. 1945;38:271-86.

3. Hippocrates. Of the epidemics. 430BC. http://classics.mit.edu/Hippocrates/ epidemics.html. Accessed 12 February 2015.

4. Stephens JWW. Blackwater fever: a historical survey and summary of observations made over a century. London: Liverpool University Press; Hodder \& Stoughton; 1937.

5. Tran TH, Day NP, Ly VC, Nguyen TH, Pham PL, Nguyen HP, Bethell DB, Dihn XS, White NJ. Blackwater fever in southern Vietnam: a prospective descriptive study of 50 cases. Clin Infect Dis. 1996;23:1274-81.

6. Bunn HF, Jandl JH. The renal handling of hemoglobin. II catabolism. J Exp Med. 1969;129:925-34.

7. Mamikonian LS, Mamo LB, Smith PB, Koo J, Lodge AJ, Turi JL. Cardiopulmonary bypass is associated with hemolysis and acute kidney injury in neonates, infants, and children. Pediatr Crit Care Med. 2014;15: e111-9.

8. Vermeulen Windsant IC, Snoeijs MG, Hanssen SJ, Altintas S, Heijmans JH, Koeppel TA, Schurink GW, Buurman WA, Jacobs MJ. Hemolysis is associated with acute kidney injury during major aortic surgery. Kidney Int. 2010;77: 913-20.

9. Janz DR, Bastarache JA, Peterson JF, Sills G, Wickersham N, May AK, Roberts 2nd LJ, Ware LB. Association between cell-free hemoglobin, acetaminophen, and mortality in patients with sepsis: an observational study. Crit Care Med. 2013:41:784-90.

10. Reeder BJ, Wilson MT. Hemoglobin and myoglobin associated oxidative stress: from molecular mechanisms to disease states. Curr Med Chem. 2005; 12:2741-51.

11. Fessel JP, Porter NA, Moore KP, Sheller JR, Roberts 2nd LJ. Discovery of lipid peroxidation products formed in vivo with a substituted tetrahydrofuran ring (isofurans) that are favored by increased oxygen tension. Proc Natl Acad Sci U S A. 2002:99:16713-8.

12. Morrow JD, Hill KE, Burk RF, Nammour TM, Badr KF, Roberts 2nd LJ. A series of prostaglandin F2-like compounds are produced in vivo in humans by a non-cyclooxygenase, free radical-catalyzed mechanism. Proc Natl Acad Sci U S A. 1990;87:9383-7.

13. Brown CD, Ghali HS, Zhao Z, Thomas LL, Friedman EA. Association of reduced red blood cell deformability and diabetic nephropathy. Kidney Int. 2005;67:295-300.

14. Hsiao LL, Howard RJ, Aikawa M, Taraschi TF. Modification of host cell membrane lipid composition by the intra-erythrocytic human malaria parasite plasmodium falciparum. Biochem J. 1991;274:121-32.

15. Billings FT, Ball SK, Roberts $\amalg$, Pretorius M. Postoperative acute kidney injury is associated with hemoglobinemia and an enhanced oxidative stress response. Free Radic Biol Med. 2011;50:1480-7.

16. Holt S, Reeder B, Wilson M, Harvey S, Morrow JD, Roberts LJ, Moore K. Increased lipid peroxidation in patients with rhabdomyolysis. Lancet. 1999; 353:1241.

17. Moore KP, Holt SG, Patel RP, Svistunenko DA, Zackert W, Goodier D, Reeder BJ, Clozel M, Anand R, Cooper CE, Morrow JD, Wilson MT, Darley-Usmar V, Roberts $L$. A causative role for Redox cycling of Myoglobin and its inhibition by Alkalinization in the pathogenesis and treatment of Rhabdomyolysis-induced renal failure. J Biol Chem. 1998;273:31731-7.

18. Charunwatthana P, Abul Faiz M, Ruangveerayut R, Maude RJ, Rahman MR, Roberts 2nd ப, Moore K, Bin Yunus E, Hoque MG, Hasan MU, Lee SJ, Pukrittayakamee S, Newton PN, White NJ, Day NP, Dondorp AM. Nacetylcysteine as adjunctive treatment in severe malaria: a randomized, double-blinded placebo-controlled clinical trial. Crit Care Med. 2009:37:516-22.

19. Das BS, Patnaik JK, Mohanty S, Mishra SK, Mohanty D, Satpathy SK, Bose TK. Plasma antioxidants and lipid peroxidation products in falciparum malaria. AmJTrop Med Hyg. 1993;49:720-5. 
20. Rubach MP, Mukemba J, Florence S, Lopansri BK, Hyland K, Volkheimer AD Yeo TW, Anstey NM, Weinberg JB, Mwaikambo ED. Impaired systemic tetrahydrobiopterin bioavailability and increased oxidized biopterins in pediatric falciparum malaria: association with disease severity. PLoS Pathog. 2015;11:e1004655.

21. Yeo TW, Lampah DA, Kenangalem E, Tjitra E, Price RN, Weinberg JB, Hyland K, Granger DL, Anstey NM. Impaired systemic tetrahydrobiopterin bioavailability and increased dihydrobiopterin in adult falciparum malaria: association with disease severity, impaired microvascular function and increased endothelial activation. PLoS Pathog. 2015;11:e1004667.

22. World Health Organization. Severe Malaria. Trop Med Int Health. 2014; 19(Suppl I).

23. Bone RC, Balk RA, Cerra FB, Dellinger RP, Fein AM, Knaus WA, Schein RM, Sibbald WJ. Definitions for sepsis and organ failure and guidelines for the use of innovative therapies in sepsis. The ACCP/SCCM consensus conference committee. American College of Chest Physicians/Society of Critical Care Medicine. Chest. 1992;101:1644-55.

24. Milne GL, Yin H, Brooks JD, Sanchez S, Jackson Roberts $\amalg$, Morrow JD. Quantification of F2-isoprostanes in biological fluids and tissues as a measure of oxidant stress. Methods Enzymol. 2007;433:113-26.

25. Waikar SS, Sabbisetti VS, Bonventre JV. Normalization of urinary biomarkers to creatinine during changes in glomerular filtration rate. Kidney Int. 2010;78:486-94.

26. Dondorp AM, Desakorn V, Pongtavornpinyo W, Sahassananda D, Silamut K Chotivanich K, Newton PN, Pitisuttithum P, Smithyman AM, White NJ, Day NP. Estimation of the total parasite biomass in acute falciparum malaria from plasma PfHRP2. PLoS Med. 2005;2:e204.

27. Dondorp AM, Angus BJ, Chotivanich K, Silamut K, Ruangveerayuth R, Hardeman MR, Kager PA, Vreeken J, White NJ. Red blood cell deformability as a predictor of anemia in severe falciparum malaria. AmJTrop Med Hyg. 1999:60:733-7.

28. Nuchsongsin F, Chotivanich K, Charunwatthana P, Omodeo-Sale F, Taramelli D, Day NP, White NJ, Dondorp AM. Effects of malaria heme products on red blood cell deformability. AmJTrop Med Hyg. 2007;77:617-22.

29. Hardeman MR, Dobbe JG, Ince C. The laser-assisted optical rotational cell analyzer (LORCA) as red blood cell aggregometer. Clin Hemorheol Microcirc. 2001;25:1-11.

30. Kidney Disease: Improving Global Outcomes (KDIGO) Acute Kidney Injury Work Group. KDIGO clinical practice guideline for Acute kidney injury. Kidney Int. Suppl 2012. 2:1-138.

31. Schwartz GJ, Munoz A, Schneider MF, Mak RH, Kaskel F, Warady BA, Furth SL. New equations to estimate GFR in children with CKD. J Am Soc Nephrol. 2009;20:629-37.

32. Staples A, LeBlond R, Watkins S, Wong C, Brandt J. Validation of the revised Schwartz estimating equation in a predominantly non-CKD population. Pediatr Nephrol. 2010;25:2321-6.

33. Zappitelli M, Parikh CR, Akcan-Arikan A, Washburn KK, Moffett BS, Goldstein SL. Ascertainment and epidemiology of acute kidney injury varies with definition interpretation. Clin J Am Soc Nephrol. 2008;3:948-54.

34. Chen S. Retooling the creatinine clearance equation to estimate kinetic GFR when the plasma creatinine is changing acutely. J Am Soc Nephrol. 2013:24:877-88

35. Boutaud O, Moore KP, Reeder BJ, Harry D, Howie AJ, Wang S, Carney CK, Masterson TS, Amin T, Wright DW, Wilson MT, Oates JA, Roberts LJ. Acetaminophen inhibits hemoprotein-catalyzed lipid peroxidation and attenuates rhabdomyolysis-induced renal failure. Proc Natl Acad Sci USA 2010;107:2699-704.

36. Spinelli SL, Lannan KL, Casey AE, Croasdell A, Curran TM, Henrichs KF, Pollock SJ, Milne GA, Refaai MA, Francis CW, Phipps RP, Blumberg N. Isoprostane and isofuran lipid mediators accumulate in stored red blood cells and influence platelet function in vitro. Transfusion. 2014;54:1569-79.

37. Morrow JD, Harris TM, Roberts LJ. Noncyclooxygenase oxidative formation of a series of novel prostaglandins: analytical ramifications for measurement of eicosanoids. Anal Biochem. 1990;184:1-10.

38. Ferreira A, Balla J, Jeney V, Balla G, Soares MP. A central role for free heme in the pathogenesis of severe malaria: the missing link? J Mol Med (Berl). 2008:86:1097-111.

39. Nguansangiam S, Day NP, Hien TT, Mai NT, Chaisri U, Riganti M, Dondorp AM, Lee SJ, Phu NH, Turner GD, White NJ, Ferguson DJ, Pongponratn E. A quantitative ultrastructural study of renal pathology in fatal plasmodium falciparum malaria. Tropical Med Int Health. 2007;12:1037-50.
40. Pfafferott C, Meiselman HJ, Hochstein P. The effect of malonyldialdehyde on erythrocyte deformability. Blood. 1982;59:12-5.

41. Pfafferott C, Nash GB, Meiselman HJ. Red blood cell deformation in shear flow. Effects of internal and external phase viscosity and of in vivo aging. Biophys J. 1985;47:695-704

42. Fairley NH, Bromfield RJ. Laboratory studies in malaria and blackwater fever. Part II. Blackwater fever. Trans R Soc Trop Med Hyg. 1934;28:141-56.

\section{Submit your next manuscript to BioMed Central and we will help you at every step:}

- We accept pre-submission inquiries

- Our selector tool helps you to find the most relevant journal

- We provide round the clock customer support

- Convenient online submission

- Thorough peer review

- Inclusion in PubMed and all major indexing services

- Maximum visibility for your research

Submit your manuscript at www.biomedcentral.com/submit
) Biomed Central 